\title{
TRAUMA RAQUIMEDULAR
}

\section{Factores predictivos de recuperación neurológica a largo plazo}

Magda Elizabeth Garzón Tarazona, MD *

\section{Resumen}

El trauma raquimedular (TRM) es el peor de aquellos a los que se logra sobrevivir. Las pérdidas en términos de independencia son catastróficas. EI TRM predomina en hombres jóvenes. ${ }^{1,2}$ Pretendemos evaluar los principales aspectos demográficos del TRM en nuestro país y establecer algunos factores predecibles de recuperación neurológica a largo plazo. ${ }^{3}$

Materiales y métodos: revisamos los registros médicos y archivos de 173 pacientes con TRM. De ellos, 131 (76\%) eran de sexo masculino y 42 (24\%) femenino; la edad promedio fue de 35,8 años.

Resultados: el tiempo que tarda en llegar el paciente al hospital fue menor de 24 horas en el 65\% de los casos; 91 tenían traumatismos en otros lugares del cuerpo. La columna cervical es el segmento más vulnerable (45,6\% de los TRM). La media de la puntuación en la escala de Glasgow fue de 15 . La presión arterial media fue de $93 \mathrm{~mm} \mathrm{Hg}$. La clasificación de la gravedad de la lesión se hizo según los criterios de la escala creada por la American Spinal Injury Association (ASIA) encontrando lesión completa, ASIA A en 40 pacientes $(23,1 \%)$, y lesiones incompletas, ASIA B en 8(4,6\%), ASIA C en 30 (17,3\%) y ASIA D en 22 $(\mathbf{1 2 \%})$. Setenta y tres pacientes no tenían ningún déficit neurológico. Once requirieron tratamiento en la unidad de cuidados intensivos (UCI), y en 19 (15\%) se siguió el protocolo de altas dosis de metilprednisolona en las primeras ocho horas postrauma. Se utilizó tracción cefálica en el $12 \%$ de los pacientes; $36 \%$ sufrieron alguna clase de complicación durante la hospitalización. La mortalidad fue de $5.2 \%$. El período de seguimiento fue de seis meses. El grado de recuperación neurológica se midió también con los criterios de la escala ASIA y fue mayor en los pacientes con lesiones incompletas. EI $60 \%$ de los pacientes con ASIA B al ingreso recuperaron un grado, el 70\% de los pacientes con ASIA C recuperaron un grado y el $\mathbf{5 0} \%$ de los pacientes ASIA D recuperaron un grado al cabo de seis meses del trauma. La disminución en el nivel de conciencia, la hipotensión y la existencia de una lesión completa desde el ingreso, condicionan una pobre recuperación neurológica a largo plazo. La presencia de complicaciones también empeora el pronóstico. Efectos benéficos en la recuperación se han encontrado en pacientes que recibieron manejo en la UCI y en aquellos que recibieron altas dosis de metilprednisolona en las primeras ocho horas.

\section{Introducción}

El TRM se define como cualquier lesión que pueda causar daño en la columna vertebral o en la médula espinal, que produzca compromiso motor, sensitivo o de los esfínteres de manera temporal o permanente..$^{1-4}$

Muchos autores han publicado estudios sobre el TRM desde la antigüedad, pues se han encontrado algunos papiros egipcios que hacen referencia a ésta clase de traumatismos. ${ }^{1}$

\footnotetext{
* Residente de Neurocirugía

** Este trabajo fue realizado bajo la tutoría del Dr. Juan Antonio Becerra, Jefe del Servicio de Neurocirugía del Hospital de San José. Profesor Titular, Fundación Universitaria de Ciencias de la Salud y del Dr. Carlos DiazGranados, Coordinador de Investigaciones Facultad de Medicina, Instructor Asociado Fundación Universitaria de Ciencias de la Salud.
}

A pesar de la implementación de nuevas medidas de seguridad y prevención, el TRM continúa siendo alto y parece ser una enfermedad de la sociedad moderna.

Cada año suceden 12.000 nuevos casos en norteamérica, 4.000 mueren antes de llegar al hospital y el 5\% durante la primera semana de hospitalización. ${ }^{4}$ La población sobreviviente aumenta cada año. La morbilidad sigue siendo alta y contribuye a empeorar el pronóstico de recuperación neurológica a largo plazo.

Hemos mejorado el tratamiento, pero es necesario evaluar cuales son los elementos del sistema que se asocian con la menor o mayor recuperación del paciente a largo plazo. 


\section{Materiales y métodos}

Usando la definición de TRM seleccionamos 380 pacientes, de los cuales excluimos aquellos cuyas condiciones podían interferir la recuperación, tales como trauma craneoencefálico severo ${ }^{5-7}$ trauma mayor en las extremidades ${ }^{8}$, antecedente de enfermedades degenerativas del sistema nervioso central y fallas graves en el seguimiento. Nuestra búsqueda reveló 173 pacientes con TRM tratados en el servicio de neurocirugía del Hospital de San José entre 1980 y 2003. Ubicamos las bases de datos institucionales y revisamos los archivos clínicos con permiso del hospital. La categorización de la gravedad de la lesión se hizo utilizando la escala ASIA. ${ }^{9}$ (Tabla 1).

Los datos clínicos alimentaron una base de datos en un computador con el programa Microsoft Access de Microsoft Corporation y el análisis estadístico se hizo mediante programas de software comerciales. El análisis multivariado se realizó según un modelo

\section{Tabla I. Escala de severidad (asia-imsop)?}

GRADOA Déficit motor y sensitivo completo, completo: incluyendo segmentos S4-S5.

GRADO B Déficit motor completo, función sensitiva incompleto: preservada hacia abajo de S4-S5.

GRADO C Función motora preservada con fuerza incompleto: muscular menor de $3 / 5$.

GRADO D Función motora preservada con fuerza incompleto: muscular por encima de $3 / 5$.

GRADO E : Normal. de regresión logística y las variables no paramétricas cualitativas y cuantitativas se analizaron mediante la prueba del Chi cuadrado y la suma de rangos de Wilcoxon. Un valor de $p$ menor o igual a 0,05 fue considerado estadísticamente significativo.

Como puede observarse (Tabla 2), a pesar de la disminución del tamaño de la muestra, las características porcentuales de la misma no presentaron variaciones importantes.

\section{Resultados}

Se estudiaron 173 pacientes. La edad promedio fue de 35,8 años, con una mediana de 33 y un rango de 9 a 104. El 76\% fueron de sexo masculino y el $24,1 \%$ femenino (relación de 4.1:1). Las causas del trauma se distribuyeron así: $30,6 \%$ caídas, $28,3 \%$ accidentes de tránsito, $25,4 \%$ violencia, $8,1 \%$ accidente laboral, $2,9 \%$ deporte y $4,6 \%$ otros. El $65,3 \%$ fue admitido al hospital antes de transcurridas 24 horas a partir del trauma; 91 con traumatismos múltiples; $45,6 \%$ presentaron un nivel de trauma cervical y $54,3 \%$ otro nivel de trauma (Tabla 3 ). El $36,4 \%$ presentó complicaciones durante su primera hospitalización. La mortalidad global del TRM fue de $5,2 \%$ de los pacientes hospitalizados.

La mediana de la escala Glasgow al ingreso fue de 15 (rango de 3 a 15). E1 42,5\% de los pacientes ingresó con un grado de severidad ASIA E (73 casos), el 23,1\% ASIA A (40) el 17,3\% ASIA C (30), el $12 \%$ ASIA D (22) y el $4,6 \%$ grado ASIA B (8).

TABLA 2. Distribución de los pacientes (en escala ASIA), en los diferentes momentos de interés.

\begin{tabular}{lccccc} 
& $\begin{array}{c}\text { ASIA } \\
\text { al ingreso }\end{array}$ & $\begin{array}{c}\text { ASIA } \\
\text { al egreso }\end{array}$ & $\begin{array}{c}\text { ASIA } \\
\text { al mes }\end{array}$ & $\begin{array}{c}\text { ASIA } \\
\text { a los 3 meses }\end{array}$ & $\begin{array}{c}\text { ASIA } \\
\text { a los 6 meses }\end{array}$ \\
\hline A & $23,1 \%$ & $17 \%$ & $15,45 \%$ & $12 \%$ & $8,5 \%$ \\
\hline B & $4,6 \%$ & $4,8 \%$ & $8,1 \%$ & $3 \%$ & $1,2 \%$ \\
\hline C & $17,3 \%$ & $16,4 \%$ & $15,4 \%$ & $16 \%$ & $14,6 \%$ \\
\hline D & $12, \%$ & $13,3 \%$ & $14,6 \%$ & $21 \%$ & $25,6 \%$ \\
\hline E & $42,2 \%$ & $48,5 \%$ & $46,3 \%$ & $48 \%$ & $50 \%$ \\
\hline$\%$ pacientes & $0,6 \%$ & $5,2 \%$ & $29 \%$ & $43 \%$ & $53 \%$ \\
no disponibles & -1 & -9 & -50 & -74 & -91
\end{tabular}


TABLA 3. Distribución de los pacientes por sexo, causa del trauma, tiempo de ingreso al Hospital, presencia de traumas múltiples y nivel anatómico del TRM.

ASIAA ASIA B ASIAC ASIAD ASIAE TOTAL

\begin{tabular}{|c|c|c|c|c|c|c|}
\hline \multicolumn{7}{|l|}{ SEXO } \\
\hline Masculino & 29 & 7 & 26 & 21 & 49 & 131 \\
\hline Femenino & 11 & 1 & 4 & 1 & 24 & 42 \\
\hline \multicolumn{7}{|l|}{ CAUSA } \\
\hline Caída & 10 & 3 & 5 & 9 & 26 & 53 \\
\hline Deporte & 1 & 0 & 0 & 0 & 4 & 5 \\
\hline Otro & 1 & 1 & 1 & 2 & 7 & 14 \\
\hline Trabajo & 2 & 1 & 2 & 2 & 7 & 14 \\
\hline A.Tránsito & 9 & 0 & 6 & 5 & 29 & 49 \\
\hline Violencia & 17 & 3 & 16 & 4 & 4 & 44 \\
\hline \multicolumn{7}{|c|}{ TIEMPO ADMISIÓN } \\
\hline $0-24$ horas & 30 & 4 & 19 & 5 & 55 & 113 \\
\hline 24-72 horas & 3 & 1 & 3 & 8 & 10 & 25 \\
\hline 72 horas -7 días & 2 & 0 & 4 & 1 & 2 & 9 \\
\hline$>7$ días & 5 & 3 & 4 & 8 & 6 & 26 \\
\hline \multicolumn{7}{|c|}{ TRAUMAS MULTIP. } \\
\hline $\mathrm{Si}$ & 18 & 5 & 18 & 17 & 50 & 108 \\
\hline No & 22 & 3 & 12 & 5 & 23 & 65 \\
\hline \multicolumn{7}{|c|}{ NIVEL DEL TRM } \\
\hline Cervical & 10 & 5 & 19 & 13 & 32 & 79 \\
\hline No cervical & 30 & 3 & 11 & 9 & 41 & 94 \\
\hline Total & 40 & 8 & 30 & 22 & 73 & 173 \\
\hline
\end{tabular}

Solo $6.4 \%$ de los pacientes requirieron manejo en la UCI (11 de 173); 85\% no recibieron corticosteroides, un $11.6 \%$ los recibieron antes de ocho horas de transcurrido el trauma. El $56.6 \%$ de los pacientes fueron sometidos a tratamiento quirúrgico, y el $43.3 \%$ recibieron únicamente manejo médico. Se utilizó tracción como parte del manejo en solo 12\%. (Tabla 4).

Los grupos con mayor grado de recuperación neurológica a los seis meses del TRM fueron los que ingresaron con severidad ASIA B,C y D. Los pacientes ASIA E llegaron sin déficit neurológico. (Tabla 5).

El $60 \%$ de los pacientes con ASIA B al ingreso ganó un grado en la escala al cabo de seis meses. E1 70\% con ASIA C al ingreso ganó un grado en la escala después de seis meses del TRM. El 50\% de los pacientes con grado ASIA D al ingreso no tenían ningún déficit neurológico (grado E en escala ASIA) a los seis meses del TRM.

El uso de corticoesteroides (en 19 pacientes) tuvo relación estadísticamente significativa (modelo de regresión logística) con el grado de recuperación neurológica a largo plazo. Disminuyó el número de pacientes con ASIA A en el transcurso de los seis meses.

La incidencia de complicaciones tuvo relación con la pobre recuperación neurológica a largo plazo; a mayor frecuencia de complicaciones peor ASIA a los seis meses.

La presión arterial media (PAM) tuvo una asociación significativa (valor de $p$ : 0,001) con el ASIA al ingreso. Así que esta variable podría constituir un factor predecible de pobre recuperación neurológica a largo plazo. 
Tabla 4. Aspectos del tratamiento

\begin{tabular}{|c|c|c|c|c|c|}
\hline & ASIA-A & ASIA-B & ASIA-C & ASIA-D & ASIA-E \\
\hline \multicolumn{6}{|c|}{ Ingreso a UCI } \\
\hline $\mathrm{Si}$ & $18 \%$ & $0 \%$ & $4 \%$ & $9 \%$ & $1 \%$ \\
\hline No & $82 \%$ & $100 \%$ & $96 \%$ & $91 \%$ & $99 \%$ \\
\hline \multicolumn{6}{|c|}{ Tipo de tratamiento } \\
\hline Quirúrgico & $57 \%$ & $75 \%$ & $70 \%$ & $68 \%$ & $49 \%$ \\
\hline Medico & $43 \%$ & $25 \%$ & $30 \%$ & $32 \%$ & $51 \%$ \\
\hline \multicolumn{6}{|c|}{ Uso de corticoides } \\
\hline $0-8$ horas & $18 \%$ & $25 \%$ & $15 \%$ & $9 \%$ & $7,5 \%$ \\
\hline $8-24$ horas & $0 \%$ & $12,5 \%$ & $7,4 \%$ & $4,5 \%$ & $1,2 \%$ \\
\hline$>24$ horas & $0 \%$ & $0 \%$ & $0 \%$ & $4,55 \%$ & $0 \%$ \\
\hline No use & $82 \%$ & $62,5 \%$ & $77,8 \%$ & $82 \%$ & $91 \%$ \\
\hline \multicolumn{6}{|c|}{ Uso tracción cefálica } \\
\hline $\mathrm{Si}$ & $3,6 \%$ & $0 \%$ & $11 \%$ & $22,7 \%$ & $12,5 \%$ \\
\hline No & $96,4 \%$ & $100 \%$ & $89 \%$ & $77,3 \%$ & $87,5 \%$ \\
\hline
\end{tabular}

Tabla 5. Grado de recuperación de los pacientes según la gravedad al ingreso.

\begin{tabular}{lc} 
ASIA AL INGRESO & PORCENTAJE \\
\hline ASIA A & $30 \%$ \\
ASIA B & $60 \%$ \\
ASIA C & $70 \%$ \\
ASIA D & $50 \%$
\end{tabular}

El nivel de conciencia medido en la escala Glasgow también tuvo una asociación significativa con el grado de severidad ASIA al ingreso (valor de $p$ de 0,002 en el modelo de regresión logística). Entre más bajo el Glasgow, más grave el daño neurológico al ingreso.

La variable más predecible del grado ASIA al egreso, al mes, a los tres y a los seis meses fue el ASIA al ingreso. Entre peor grado ASIA al ingreso, también será peor ASIA a los seis meses y entre mejor ASIA al ingreso, mejor ASIA a los seis meses.
La presencia de politraumatismos y el nivel anatómico de la lesión no tuvieron ninguna asociación significativa con el grado de daño neurológico al ingreso.

De las variables cualitativas, el tipo de tratamiento y el uso o no de tracción cefálica, no tuvieron asociación estadísticamente significativa con el grado ASIA durante el seguimiento. (Tabla 6).

\section{Discusión}

Nuestro estudio coincide con reportes previos realizados usando otras escalas de medición. Sin embargo, no pudimos demostrar que todas las variables seleccionadas tuvieran relación con el grado de recuperación neurológica.

Algunos de los buenos resultados en términos de recuperación a largo plazo pueden deberse a los grandes avances en el diagnóstico y manejo tanto pre como hospitalario. Las fallas en el seguimiento

\begin{tabular}{lcccc}
\multicolumn{5}{c}{ TABLA 6. Valor de $p$ de las variables estadísticamente relacionadas } \\
con el grado de recuperación a lo largo de seis meses.
\end{tabular}


pueden ser objeto de debate considerando las limitaciones propias de los análisis retrospectivos.

\section{Conclusiones}

Establecimos que el deterioro en el nivel de conciencia, la hipotensión y la presencia de una lesión completa (medida mediante la escala ASIA) son factores predictivos de pobre recuperación neurológica a largo plazo, coincidiendo con los estudios realizados por Burns ${ }^{10}$, Geisler ${ }^{11}$, Tator ${ }^{12}$ y Zach ${ }^{13}$. La presencia de complicaciones también disminuye las expectativas de recuperación como lo demostró Vijaj A. ${ }^{14}$ en sus publicaciones.

El tratamiento en la UCI y el uso del protocolo de altas dosis de metilprednisolona en las primeras ocho horas incrementan el grado de recuperación funcional a largo plazo ${ }^{15-18}$.

No pudimos demostrar que el tiempo que tarda el paciente en ingresar al hospital, la presencia de múltiples traumas, el nivel anatómico del trauma, el tipo de tratamiento o el uso de tracción cefálica, tengan alguna incidencia sobre el grado de recuperación neurológica a largo plazo en pacientes con TRM..$^{19-22}$

\section{Referencias}

1. Benzel EC, Tator CH. Contemporary Managemente of Spinal Cord Injury.AANS Publications Committee, Park Ridge, Il. 1985.

2. Stripling TE. The cost of economic consequences of spinal cord injury. Paraplegia News 1990; 50-54.

3. Burns A, Ditunno J. Establishing Prognosis and Maximizing Functional Outcomes After Spinal Cord Injury. Spine 2001; 26(24):S137-S145.

4. DeVivo MJ, Go BK, Jackson AB. Overview of the national spinal cord injury statistical center database. J Spinal Cord Med. 2002 25(4):335-8.

5. Michael DB, Guyot DR, Darmody WR. Coincidence of head and cervical spine injury. J Neurotrauma 1989; 6: 177 - 89.

6. Kraus JF. Injury to the head and spinal cord: The epidemiological relevance of the medical literature published from 1960 to 1978 . J Neurosurgery. 1980; 18: $324-30$.
7. Graus JF. Injury to the Head and Spinal Cord: The epidemiological relevance of the medical literature published from 1960 - 1978. J Neurosurg 1980; 53: S-S10.

8. Duker TB, Saul TG, The poly-trauma and spinal cord injury. In: Tator CH, ed. Early Management of Acute Spinal Cord Injury. New York: Raven; 1982 p $53-8$.

9. American Spinal Injury Association; International Medical Society or Paraplegia. International standards for Neurological And Functional Classification of Spinal Cord Injury. Revised, 1992. Chicago, Ill: ASIA/IMSOP; 1992.

10. Burns A, Ditunno J. Establishing Prognosis and Maximizing Functional Outcomes After Spinal Cord Injury. Spine 2001; supplement; 26 (24):S137-S145.

11. Geisler F, Coleman W, Grieco G, et al. Measurements and Recovery Patterns in a Multicenter Study of Acute Spinal Cord Injury. Spine 2001; supplement ; 26(24):S68-S86.

12. Tator CH, Rowed DW, Schwartz ML. Sunnybrook cord injury scales for assessing neurological Injury and neurological recovery. In: Tator CH, ed. Early Management of Acute Spinal Cord Injury. New York: Raven 1982; 2:7-24.

13. Zach GA, Séller W, Dollfus P. Treatment results of spinal cord injuries in thewiss Paraplegic Centre of Basel. Paraplegia 1976; 14: 58-65.

14. Vijai PR, Suys S, Villanueva P. Prevention and treatment of medical complications. In Tator $\mathrm{CH}$, ed. Early Management of Acute Spinal Cord Injury. New York; 1982; 2:7-24.

15. Apuzzo Michael LJ. Pharmacological Therapy alter Acute Cervical Spinal Cord Injury. Neurosurgery; 2002; 50 (3): S63-S72.

16. Michael G. Fehlings. Editorial: Recomendations Regarding the Use of Methylprednidolone in Acute Spinal Cord Injury. Spine 2001;26(24):S56-S57.

17. Pagliacci M, Celan M, Zampolini M, et al. An Italian survey of traumatic spinal cord injury. The Gruppo Italiano Studio Epidemiologico Mielolesioni study. Archives of Physical Medicine and Rehabilitation. 2003; 84 (9):1266-75.

18. Short DJ, Masry WS, Jones PW. High dose methylprednisolone in the management of acute spinal cord injury: A systematic review from a clinical perspective. Spinal Cord 2000; 38: 273-86.

19. Guidelines for Management of Acute Spinal Cord Injury. Inicial Closed Reduction of Cervical Spine Fracture-Dislocation Injuries. Neurosurgery; 2002; 50 (3): S44-S45.

20. Fehlings M Sekhon L, Tator CH. The role and timing of Decompression in Acute Spinal Cord Injury. Spine 2001; 26 (24):S101-S110.

21. Frankel HL, The value of postural reduction in the initial management of closed injuries of the spine with paraplegia and tetraplegia. Paraplegia 1969; 7: 179-192.

22. Russ P. Nockels. Nonoperative Management of Acute Spinal Cord Injury. Spine 2001; 26 (24):S31-S37. 